\title{
A Graph Theoretic Method to Find Decentralized Fixed Modes of LTI Systems
}

\author{
Javad Lavaei and Amir G. Aghdam \\ Department of Electrical and Computer Engineering, Concordia University \\ Montréal, QC Canada H3G 1 M8 \\ \{j_lavaei,aghdam\}@ece.concordia.ca
}

\begin{abstract}
This paper deals with the decentralized control of systems with distinct modes. A simple graph-theoretic based approach is proposed to identify those modes of the system which cannot be moved by means of a linear time-invariant decentralized controller. First, the system is transformed into its Jordan state-space representation. Then, a matrix is computed, which has the same order as the transfer function matrix of the system. A bipartite graph is constructed accordingly in terms of the computed matrix. Now, the problem of identifying the DFMs of the system reduces to verifying if this graph has a complete bipartite subgraph with a certain property. The proposed approach is quite simpler than the existing ones, which often require calculating the rank of a huge number of matrices.
\end{abstract}

\section{Introduction}

Numerous real-world systems can be envisaged as the interconnected systems consisting of a number of subsystems. Usually, the desirable control structure for this class of systems is decentralized, which comprises a set of local controllers for the subsystems (Wang and Davison, 1973; Davison and Chang, 1990; Lavaei and Aghdam (a), 2006). Decentralized control system theory has found applications in large space structure, communication networks, power systems, etc. (Inalhan, Stipanovic and Tomlin, 2002; Lavaei, Momeni and Aghdam, 2006;

\footnotetext{
1 This work has been supported by the Natural Sciences and Engineering Research Council of Canada under grant RGPIN-262127-03.
}

Lavaei and Aghdam (b), 2006).

The notion of decentralized fixed mode (DFM) was introduced in (Wang and Davison, 1973), where it was shown that any mode of a system which is not a DFM can be placed freely everywhere in the complex plane by means of a linear time-invariant (LTI) controller. An algebraic characterization of DFMs was presented in (Anderson and Clements, 1981). A method was then proposed in (Anderson et al., 1982) to characterize the DFMs of a system in terms of its transfer function. It was shown in (Davison and Wang, 1985) that the DFMs of any system can be attained by computing the transmission zeros of a set of systems derived from the original system. In (Davison and Chang, 1990), an algorithm was 
presented to identify the DFMs of the system by checking the rank of a set of matrices. It is worth noting that the number of the systems whose transmission zeros need to be checked in (Davison and Wang, 1985) and the number of matrices whose ranks are to be computed in (Davison and Chang, 1990) depend exponentially on the number of the subsystems of the original system. This means that while these methods are theoretically applicable to any multi-input multi-output (MIMO) system, they are computationally ill-conditioned. The method introduced in (Gong and Aldeen, 1992) addresses this practical problem by partitioning the system into a number of modified subsystems, obtained based on the strong connectivity of the system's graph. Then, instead of finding the DFMs of the original system, one can compute the DFMs of the modified subsystems to reduce the corresponding computational complexity. However, the computational burden can still be high when the system consists of several strongly connected subsystems. In general, the method given in (Gong and Aldeen, 1992) is more effective for medium-sized systems, while the one in (Davison and Chang, 1990) is only appropriate for small-sized systems. It is to be noted that the method introduced in (Davison and Chang, 1990) is widely used in the literature for the characterization of the DFMs.

This paper aims to present a quite simple approach to find the DFMs of a system with distinct modes. To this end, a matrix is obtained first, which resembles the transfer function matrix of the system at one point. Then, a bipartite graph is constructed in terms of this matrix. It is shown that having a complete bipartite subgraph with a certain property is equivalent to having a DFM. The combinatorial approach proposed in the present paper is substantially simpler than the conventional methods for finding the DFMs. The efficacy of the proposed method is demonstrated in a numerical example.
This paper is organized as follows. The problem formulation and main development are presented in Section II, which are followed by a numerical example in Section III. Some concluding remarks are then given in Section IV.

\section{Main results}

Consider a LTI interconnected system $\mathcal{S}$ consisting of $\nu$ subsystems $S_{1}, S_{2}, \ldots, S_{\nu}$, represented by:

$$
\begin{aligned}
\dot{x}(t) & =A x(t)+\sum_{j=1}^{\nu} B_{j} u_{j}(t) \\
y_{i}(t) & =C_{i} x(t)+\sum_{j=1}^{\nu} D_{i j} u_{j}(t), \quad i \in \bar{\nu}:=\{1,2, \ldots, \nu\}
\end{aligned}
$$

where $x(t) \in \Re^{n}$ is the state, and $u_{i}(t) \in \Re^{m_{i}}$ and $y_{i}(t) \in \Re^{r_{i}}, i \in \bar{\nu}$, are the input and the output of the $i^{\text {th }}$ subsystem, respectively. Assume that each subsystem is desired to be controlled by a local controller. The $i^{\text {th }}$ local controller observes only the local output $y_{i}(t)$ to construct the local input $u_{i}(t)$ of the $i^{\text {th }}$ subsystem, $i \in \bar{\nu}$. Throughout this paper, the term "decentralized controller" is referred to the union of all local controllers. It is desired now to find the DFMs of the system $\mathcal{S}$, if any.

Suppose the eigenvalues of $A$ are distinct. Write the matrix $A$ as $T \mathbf{A} T^{-1}$, where $T$ is the eigenvector matrix of $A$. Denote the matrix $\mathbf{A}$ as follows:

$$
\mathbf{A}=\left[\begin{array}{cccc}
\sigma_{1} & 0 & \cdots & 0 \\
0 & \sigma_{2} & \cdots & 0 \\
\vdots & \cdots & \ddots & \vdots \\
0 & 0 & \cdots & \sigma_{n}
\end{array}\right]
$$

where $\sigma_{i}, i \in\{1,2, \ldots, n\}$ denote the modes of the system $\mathcal{S}$. Therefore, the system $\mathcal{S}$ can be represented in the 
decoupled form as:

$$
\begin{aligned}
\dot{\mathbf{x}}(t) & =\mathbf{A} \mathbf{x}(t)+\sum_{j=1}^{\nu} \mathbf{B}_{j} u_{j}(t) \\
y_{i}(t) & =\mathbf{C}_{i} \mathbf{x}(t)+\sum_{j=1}^{\nu} \mathbf{D}_{i j} u_{j}(t), \quad i \in \bar{\nu}
\end{aligned}
$$

where:

$$
\begin{aligned}
{\left[\begin{array}{lll}
\mathbf{B}_{1} \cdots & \mathbf{B}_{\nu}
\end{array}\right] } & =T^{-1}\left[\begin{array}{lll}
B_{1} & \cdots & B_{\nu}
\end{array}\right], \\
{\left[\begin{array}{ccc}
\mathbf{C}_{1} \cdots & \mathbf{C}_{\nu}
\end{array}\right] } & =\left[\begin{array}{lll}
C_{1} \cdots & C_{\nu}
\end{array}\right] T, \\
\mathbf{D}_{i j} & =D_{i j}, \quad i, j \in \bar{\nu}
\end{aligned}
$$

NOtation: For any $i, j \in \bar{\nu}$ :

- Denote the $\left(\mu_{1}, \mu_{2}\right)$ entry of $\mathbf{B}_{i}$ with $\mathbf{b}_{i}^{\mu_{1}, \mu_{2}}$, for any $1 \leq \mu_{1} \leq n, 1 \leq \mu_{2} \leq m_{i}$.

- Denote the $\left(\mu_{1}, \mu_{2}\right)$ entry of $\mathbf{C}_{i}$ with $\mathbf{c}_{i}^{\mu_{1}, \mu_{2}}$, for any $1 \leq \mu_{1} \leq r_{i}, 1 \leq \mu_{2} \leq n$.

- Denote the $\left(\mu_{1}, \mu_{2}\right)$ entry of $\mathbf{D}_{i j}$ with $\mathbf{d}_{i j}^{\mu_{1}, \mu_{2}}$, for any $1 \leq \mu_{1} \leq r_{i}, 1 \leq \mu_{2} \leq m_{j}$.

The following theorem formulates the DFMs of the system $\mathcal{S}$.

Theorem 1 Assume that the mode $\sigma_{i}, i \in\{1,2, \ldots, n\}$, is controllable as well as observable. $\sigma_{i}$ is a DFM of the system $\mathcal{S}, \nu \geq 2$, if and only if there exist a permutation of $\{1,2, \ldots, \nu\}$ denoted by distinct integers $i_{1}, i_{2}, \ldots, i_{\nu}$ and an integer $p \in[1, \nu-1]$ such that $\mathbf{b}_{\eta}^{i, \alpha}=\mathbf{c}_{\gamma}^{\beta, i}=0$, and:

$$
\sum_{\mu=1, \mu \neq i}^{n} \frac{\mathbf{b}_{\eta}^{\mu, \alpha} \mathbf{c}_{\gamma}^{\beta, \mu}}{\sigma_{\mu}-\sigma_{i}}=\mathbf{d}_{\gamma \eta}^{\beta, \alpha}
$$

for all $\eta, \gamma, \alpha$ and $\beta$ given by:

$$
\begin{aligned}
& \eta \in\left\{i_{1}, i_{2}, \ldots, i_{p}\right\}, \quad \gamma \in\left\{i_{p+1}, i_{p+2}, \ldots, i_{\nu}\right\} \\
& 1 \leq \alpha \leq m_{\eta}, \quad 1 \leq \beta \leq r_{\gamma}
\end{aligned}
$$

Proof: It is known that $\sigma_{i}$ is a DFM of the system $\mathcal{S}\left(S_{1}, S_{2}, \ldots, S_{\nu}\right)$ if and only if there exist a permutation of $\{1,2, \ldots, \nu\}$ denoted by distinct integers $i_{1}, i_{2}, \ldots, i_{\nu}$ and an integer $p \in[0, \nu]$ such that the rank of the following matrix is less than $n$ (Davison and Chang, 1990):

$$
\left[\begin{array}{ccccc}
\mathbf{A}-\sigma_{i} I_{n} & \mathbf{B}_{i_{1}} & \mathbf{B}_{i_{2}} & \ldots & \mathbf{B}_{i_{p}} \\
\mathbf{C}_{i_{p+1}} & \mathbf{D}_{i_{p+1} i_{1}} & \mathbf{D}_{i_{p+1} i_{2}} & \ldots & \mathbf{D}_{i_{p+1} i_{p}} \\
\mathbf{C}_{i_{p+2}} & \mathbf{D}_{i_{p+2} i_{1}} & \mathbf{D}_{i_{p+2} i_{2}} & \ldots & \mathbf{D}_{i_{p+2} i_{p}} \\
\vdots & \vdots & \vdots & \ddots & \vdots \\
\mathbf{C}_{i_{\nu}} & \mathbf{D}_{i_{\nu} i_{1}} & \mathbf{D}_{i_{\nu} i_{2}} & \ldots & \mathbf{D}_{i_{\nu} i_{p}}
\end{array}\right]
$$

In addition, since it is assumed that the mode $\sigma_{i}$ is controllable and observable, the rank of the matrix (7) is equal to $n$ for $p=0$ and $p=\nu$. Therefore, the condition $0 \leq p \leq \nu$ given above can be reduced to $1 \leq p \leq \nu-1$. It is clear that the rank of the matrix $\mathbf{A}-\sigma_{i} I_{n}$ is $n-1$, and also, the $i^{\text {th }}$ column and the $i^{\text {th }}$ row of this matrix are both zeros. Hence, if there exists a nonzero entry either in the $i^{\text {th }}$ column or in the $i^{\text {th }}$ row of the matrix given in (7), its rank will be at least $n$. As a result, the rank of the matrix in (7) is less than $n$, if and only if both of the following conditions hold:

i) All of the entries of the $i^{\text {th }}$ column and the $i^{\text {th }}$ row of the matrix given in (7) are zero, i.e., $\mathbf{b}_{\eta}^{i, \alpha}=\mathbf{c}_{\gamma}^{\beta, i}=0$ for any $\alpha, \beta, \eta$, and $\gamma$ satisfying (6). 
ii) The rank of the following matrix:

$$
\left[\begin{array}{ccccccc}
\sigma_{1}^{i} & \ldots & 0 & 0 & \ldots & 0 & \mathbf{b}_{\eta}^{1, \alpha} \\
\vdots & \ddots & \vdots & \vdots & \ddots & \vdots & \vdots \\
0 & \ldots & \sigma_{i-1}^{i} & 0 & \ldots & 0 & \mathbf{b}_{\eta}^{i-1, \alpha} \\
0 & \ldots & 0 & \sigma_{i+1}^{i} & \ldots & 0 & \mathbf{b}_{\eta}^{i+1, \alpha} \\
\vdots & \ddots & \vdots & \vdots & \ddots & \vdots & \vdots \\
0 & \ldots & 0 & 0 & \ldots & \sigma_{n}^{i} & \mathbf{b}_{\eta}^{n, \alpha} \\
\mathbf{c}_{\gamma}^{\beta, 1} & \ldots & \mathbf{c}_{\gamma}^{\beta, i-1} & \mathbf{c}_{\gamma}^{\beta, i+1} & \ldots & \mathbf{c}_{\gamma}^{\beta, n} & \mathbf{d}_{\gamma \eta}^{\beta, \alpha}
\end{array}\right]
$$

(which is a sub-matrix of the one given by (7)) is less than $n$ for any $\alpha, \beta, \eta$, and $\gamma$ satisfying (6), where $\sigma_{j}^{i}:=\sigma_{j}-\sigma_{i}, i, j \in\{1,2, \ldots, n\}$. Partition the matrix given by (8) into four sub-matrices, and denote it with $\left[\begin{array}{cc}A_{i} & \Phi_{1} \\ \Phi_{2} & \mathbf{d}_{\gamma \eta}^{\beta, \alpha}\end{array}\right]$, where $A_{i} \in \Re^{(n-1) \times(n-1)}, \Phi_{1} \in$ $\Re^{(n-1) \times 1}$, and $\Phi_{2} \in \Re^{1 \times(n-1)}$. Since the matrix $A_{i}$ is nonsingular (because it is assumed that $\sigma_{1}, \ldots, \sigma_{n}$ are distinct), one can write:

$\operatorname{det}\left[\begin{array}{cc}A_{i} & \Phi_{1} \\ \Phi_{2} & \mathbf{d}_{\gamma \eta}^{\beta, \alpha}\end{array}\right]=\operatorname{det}\left(A_{i}\right) \times \operatorname{det}\left(\mathbf{d}_{\gamma \eta}^{\beta, \alpha}-\Phi_{2} A_{i}^{-1} \Phi_{1}\right)$

Thus, the rank of the matrix given in (8) is less than $n$, if and only if the scalar $\Phi_{2} A_{i}^{-1} \Phi_{1}$ is equal to $\mathbf{d}_{\gamma \eta}^{\beta, \alpha}$, i.e.:

$$
\sum_{\mu=1, \mu \neq i}^{n} \frac{\mathbf{b}_{\eta}^{\mu, \alpha} \mathbf{c}_{\gamma}^{\beta, \mu}}{\sigma_{\mu}^{i}}=\mathbf{d}_{\gamma \eta}^{\beta, \alpha}
$$

Define now the matrix $M_{i}$ as:

$$
\begin{aligned}
M_{i}:= & \mathbf{C} \times \operatorname{diag}\left(\left[\frac{1}{\sigma_{1}-\sigma_{i}}, \ldots, \frac{1}{\sigma_{i-1}-\sigma_{i}}, 0,\right.\right. \\
& \left.\left.\frac{1}{\sigma_{i+1}-\sigma_{i}}, \ldots, \frac{1}{\sigma_{\nu}-\sigma_{i}}\right]\right) \mathbf{B}-\mathbf{D}
\end{aligned}
$$

and denote its $\left(\mu_{1}, \mu_{2}\right)$ block entry with $M_{i}^{\mu_{1}, \mu_{2}} \in$ $\Re^{r_{\mu_{1}} \times m_{\mu_{2}}}$, for any $\mu_{1}, \mu_{2} \in \bar{\nu}$. Note that the expression of $M_{i}$ resembles that of the transfer function matrix of the system $\mathcal{S}$, while the sign of $\mathbf{D}$ is different in $M_{i}$.

Theorem 2 The mode $\sigma_{i}, i \in\{1,2, \ldots, n\}$, is a DFM of the system $\mathcal{S}, \nu \geq 2$, if and only if any of the following conditions holds:

(i) The $i^{\text {th }}$ row of the matrices $\mathbf{B}_{1}, \mathbf{B}_{2}, \ldots, \mathbf{B}_{\nu}$ are zero.

ii) The $i^{\text {th }}$ column of the matrices $\mathbf{C}_{1}, \mathbf{C}_{2}, \ldots, \mathbf{C}_{\nu}$ are zero.

iii) There exist a permutation of $\{1,2, \ldots, \nu\}$ denoted by distinct integers $i_{1}, i_{2}, \ldots, i_{\nu}$ and an integer $p \in[1, \nu-1]$ such that $M_{i}^{\gamma, \eta}$ is a zero matrix for any $\eta \in\left\{i_{1}, i_{2}, \ldots, i_{p}\right\}$ and $\gamma \in\left\{i_{p+1}, i_{p+2}, \ldots, i_{\nu}\right\}$, and moreover the $i^{\text {th }}$ row of the matrices $\mathbf{B}_{1}, \mathbf{B}_{2}, \ldots ., \mathbf{B}_{i_{p}}$ and the $i^{\text {th }}$ column of $\mathbf{C}_{i_{p+1}}, \mathbf{C}_{i_{p+2}}, \ldots ., \mathbf{C}_{i_{\nu}}$ are all zero.

Proof: Criteria (i) and (ii) are equivalent to the uncontrollability and the unobservability, respectively. Furthermore, Criterion (iii) is resulted from Theorem 1, on noting that $M_{i}^{\gamma, \eta}$ is a $r_{\gamma} \times m_{\eta}$ matrix whose $(\beta, \alpha)$ entry is equal to:

$$
\sum_{\mu=1, \mu \neq i}^{n} \frac{\mathbf{b}_{\eta}^{\mu, \alpha} \mathbf{c}_{\gamma}^{\beta, \mu}}{\sigma_{\mu}-\sigma_{i}}-\mathbf{d}_{\gamma \eta}^{\beta, \alpha}
$$

for any $\beta \in\left[1, r_{\gamma}\right], \alpha \in\left[1, m_{\eta}\right]$.

It is desired now to construct a graph based on the matrix $M_{i}$. Consider a bipartite graph $\mathcal{G}_{i}$ with $\nu$ vertices $1,2, \ldots, \nu$ in each of its vertex sets, namely set 1 and set 2 . For any $\mu_{1}, \mu_{2} \in \bar{\nu}$, connect vertex $\mu_{1}$ of set 1 to vertex $\mu_{2}$ of set 2 if the matrix $M_{i}^{\mu_{1}, \mu_{2}}$ is a zero matrix. Then, mark vertex $\mu_{1}$ of set 1 if the $i^{\text {th }}$ column of the matrix 
$C_{\mu_{1}}$ is a zero vector, for any $\mu_{1} \in \bar{\nu}$. Likewise, mark vertex $\mu_{2}$ of set 2 if the $i^{\text {th }}$ row of the matrix $B_{\mu_{2}}$ is a zero vector, for any $\mu_{2} \in \bar{\nu}$.

The following algorithm results from Theorem 2 for verifying whether or not the mode $\sigma_{i}$ is a DFM of the system $\mathcal{S}$

\section{Algorithm 1:}

Step 1) Compute the matrix $M_{i}$, and construct the graph $\mathcal{G}_{i}$ in terms of it, as pointed out earlier.

Step 2) Verify if all of the vertices in set 1 of the graph $\mathcal{G}_{i}$ are marked. If yes, go to Step 6.

Step 3) Verify if all of the vertices in set 2 of the graph $\mathcal{G}_{i}$ are marked. If yes, go to Step 6.

Step 4) Check whether the graph $\mathcal{G}_{i}$ includes a complete bipartite subgraph such that all of its vertices are marked and moreover the set of the indices of its vertices is equal to the set $\bar{\nu}$. If yes, go to Step 6 .

Step 5) The mode $\sigma_{i}$ is not a DFM of the system $\mathcal{S}$. Stop the algorithm.

Step 6) The mode $\sigma_{i}$ is a DFM of the system $\mathcal{S}$. Stop the algorithm.

Algorithm 1 proposes a simple graph-theoretic approach to find the DFMs of the system $\mathcal{S}$. This method requires deriving a certain matrix, and then checking the existence of a complete subgraph in a graph, which can be accomplished using numerous efficient algorithms. In contrast, the existing methods require the rank of several matrices (say $2^{\nu}$ ) to be checked, which can be cumbersome when the matrix is of high dimension. In fact, the above algorithm presents a simple combinatorial procedure as a more efficient alternative to find the DFMs of a system (with distinct modes).

Corollary $\mathbf{1}$ Denote the number of matrices $\mathbf{B}_{1}, \mathbf{B}_{2}, \ldots, \mathbf{B}_{\nu}$ whose $i^{\text {th }}$ row are zero with $\Gamma_{i}$. Furthermore, denote the number of matrices $\mathbf{C}_{1}, \mathbf{C}_{2}, \ldots, \mathbf{C}_{\nu}$ whose $i^{\text {th }}$ column are zero with $\bar{\Gamma}_{i}$. If $\Gamma_{i}+\bar{\Gamma}_{i}$ is less than $\nu$, then $\sigma_{i}$ is not a $D F M$ of the system $\mathcal{S}$.

Proof: It is straightforward to show that if $\Gamma_{i}+\bar{\Gamma}_{i}$ is less than $\nu$, none of Steps 1, 2 or 3 of Algorithm 1 is fulfilled.

Corollary 1 presents a quite simple test as a sufficient condition to verify whether $\sigma_{i}$ can be a DFM of the system or not.

\section{Illustrative example}

Consider the system $\mathcal{S}$ consisting of five single-input single-output (SISO) subsystems with the following 
state-space matrices:

$$
\begin{aligned}
& \mathbf{A}=\operatorname{diag}([1,2,3,4,5]), \\
& \mathbf{B}=\left[\begin{array}{ccccc}
0 & 0 & 0 & 1 & 2 \\
2 & 1 & 3 & 1 & 4 \\
0 & 2 & 4 & -1 & 5 \\
0 & 0 & 3 & 0 & -3 \\
0 & 0 & 0 & 3 & -1
\end{array}\right] \text {, } \\
& \mathbf{C}=\left[\begin{array}{ccccc}
0 & 3 & 2 & 1 & 4 \\
0 & 3 & 4 & 2 & -1 \\
5 & 4 & 3 & -2 & 4 \\
0 & 2 & 3 & 1 & 3 \\
0 & -2 & -3 & -2 & -4
\end{array}\right] \text {, } \\
& \mathbf{D}=\left[\begin{array}{ccccc}
6 & 5 & 14 & 3 & 2 \\
6 & 7 & 19 & 4 & 2 \\
8 & 7 & 16 & -2 & -4 \\
4 & 5 & 13 & 0 & 1 \\
-4 & -5 & -14 & -1 & 2
\end{array}\right]
\end{aligned}
$$

It is desired to verify which of the modes $\sigma_{i}=i, i \in \bar{\nu}=$ $\{1,2,3,4,5\}$, are DFMs of the system $\mathcal{S}$. First, let the test given in Corollary 1 be carried out. Since the first entries of $\mathbf{B}_{1}, \mathbf{B}_{2}, \mathbf{B}_{3}, \mathbf{C}_{1}, \mathbf{C}_{2}, \mathbf{C}_{4}$ and $\mathbf{C}_{5}$ are all zero, $\Gamma_{1}+\bar{\Gamma}_{1}$ is equal to 7 . Similarly, one can conclude that:

$\Gamma_{2}+\bar{\Gamma}_{2}=0, \quad \Gamma_{3}+\bar{\Gamma}_{3}=1, \quad \Gamma_{4}+\bar{\Gamma}_{4}=3, \quad \Gamma_{5}+\bar{\Gamma}_{5}=3$

Due to the fact that $\Gamma_{i}+\bar{\Gamma}_{i}<5$ for $i=2,3,4,5$, it follows from Corollary 1 that none of the modes 2,3,4 and 5 is a DFM of the system $\mathcal{S}$. Algorithm 1 will now be used to find out whether $\sigma_{1}=1$ is a DFM. The matrix $M_{1}$ will be obtained as:

$$
\begin{aligned}
M_{1}= & \mathbf{C} \times \operatorname{diag}\left(\left[0,1, \frac{1}{2}, \frac{1}{3}, \frac{1}{4}\right]\right) \mathbf{B}-\mathbf{D} \\
= & {\left[\begin{array}{ccccc}
0 & 0 & 0 & 2 & 13 \\
0 & 0 & 0 & -3.75 & 18.25 \\
0 & 0 & 0 & 7.5 & 28.5 \\
0 & 0 & 0 & 2.75 & 12.75 \\
0 & 0 & 0 & -2.5 & -14.5
\end{array}\right] }
\end{aligned}
$$

The graph $\mathcal{G}_{1}$ corresponding to the matrix $M_{1}$ is sketched in Figure 1. Since the first entries of $\mathbf{B}_{1}, \mathbf{B}_{2}, \mathbf{B}_{3}, \mathbf{C}_{1}, \mathbf{C}_{2}, \mathbf{C}_{4}$ and $\mathbf{C}_{5}$ are all zero, vertices 1,2 and 3 from set 2, and vertices 1, 2, 4 and 5 from set 1 of the graph $\mathcal{G}_{1}$ are marked by filled circles, as shown in the figure. It can be easily observed that vertices 4,5 of set 1 and vertices 1, 2, 3 of set 2 fulfill the following criteria:

- All of them are marked.

- They constitute a complete bipartite graph.

- The set of their labels is equal to $\bar{\nu}$.

Therefore, $\sigma_{1}=1$ is a DFM of the system (from Step 3 of Algorithm 1).

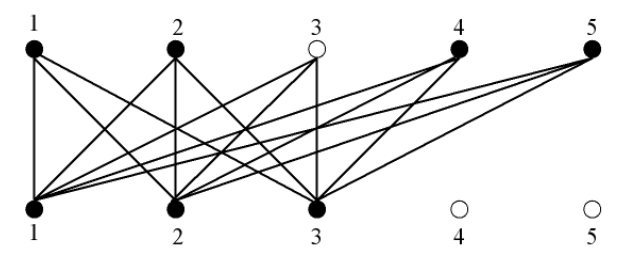

Fig. 1. The graph $\mathcal{G}_{1}$ corresponding to the matrix $M_{1}$ given in (15).

Regarding the mode $\sigma_{3}=3$, let Algorithm 1 be pursued for this mode regardless of the observation that it failed 
the test given in Corollary 1. The matrix $M_{3}$ is equal to:

$$
\begin{aligned}
M_{3}= & \mathbf{C} \times \operatorname{diag}\left(\left[-1,0,1, \frac{1}{2}, \frac{1}{3}\right]\right) \mathbf{B}-\mathbf{D} \\
= & {\left[\begin{array}{ccccc}
-12 & -8 & -20 & 0 & -19 \\
-12 & -10 & -22 & -8.5 & -19 \\
-16 & -11 & -34 & 1.5 & -13 \\
-8 & -7 & -16 & 2.5 & -13 \\
8 & 7 & 14 & -3 & 14
\end{array}\right] }
\end{aligned}
$$

The corresponding graph $\mathcal{G}_{3}$ is depicted in Figure 2. Since there are not enough edges in the graph to create a complete bipartite subgraph which spans all the indices, thus $\sigma_{3}=3$ is not a DFM of the system (which also confirms the result obtained from Corollary 1).

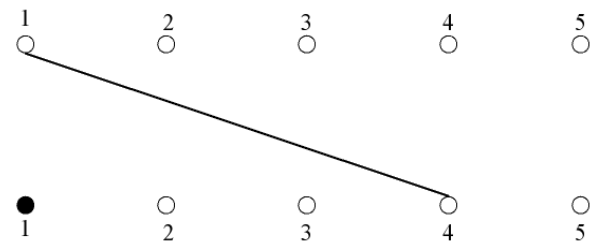

Fig. 2. The graph $\mathcal{G}_{3}$ corresponding to the matrix $M_{3}$ given in (16).

Consequently, the system has only one DFM at 1.This result could also be obtained by using the method given in (Davison and Chang, 1990) or (Davison and Wang, 1985), which require the rank of $5 \times 2^{5}$ matrices with the dimensions between 5 and 10 be checked. The sizable difference between the computational costs required by the method presented in this paper and the ones given in (Davison and Chang, 1990; Davison and Wang, 1985) demonstrates the efficacy of this work. It is worth mentioning that the results obtained here by using the proposed method are attained by hand, while the methods given in (Davison and Chang, 1990; Davison and Wang, 1985) require a proper software (such as MATLAB) to solve the problem.

\section{Conclusions}

This paper aims to find the modes of a system (with distinct modes) which are fixed with respect to any LTI decentralized controller. Unlike the existing methods which require the computation of the rank of numerous matrices, the approach proposed here transforms the knowledge of the system into a bipartite graph. Then, it is shown that a mode is decentrally fixed if there exists a complete bipartite subgraph with a certain property. The efficacy of the proposed method is demonstrated in a numerical example.

\section{References}

Anderson, B. L. O. (1982). Transfer function matrix description of decentralized fixed modes. IEEE Transactions on Automatic Control. 27(6), 1176-1182.

Anderson, B. L. O., \& Clements, D. J. (1981). Algebraic characterizations of fixed modes in decentralized systems. Automatica. 17(5), 703-712.

Davison, E. J., \& Chang, T. N. (1990). Decentralized stabilization and pole assignment for general proper systems. IEEE Transactions on Automatic Control.

Davison, E. J., \& Wang, S. H. (1985). A characterization of decentralized fixed modes in terms of transmission zeros. IEEE Transactions on Automatic Control. 30(1), 81-82. 35(6), 652-664.

Inalhan, G., Stipanovic, D. M., \& Tomlin, C. J. (2002). Decentralized optimization with application to multiple aircraft coordination, Proceedings of the 41 st IEEE Conference on Decision and Control. Vegas, NV. (pp. 1147-1155).

Gong, Z., \& Aldeen, M. (1992). On the characterization of fixed modes in decentralized control. IEEE Transactions on Automatic Control. 37(7), 10461050. 
Lavaei, J., \& Aghdam, A. G. (2006). Elimination of fixed modes by means of high-performance constrained periodic control, to appear in Proceedings of the 45th IEEE Conference on Decision and Control. San Diego, CA.

Lavaei, J., \& Aghdam, A. G. (2006). Decentralized control design for interconnected systems based on a centralized reference controller, to appear in Proceedings of the 45th IEEE Conference on Decision and Control. San Diego, CA.

Lavaei, J., Momeni, A., \& Aghdam, A. G. (2006). HighPerformance decentralized control for formation flying with leader-follower structure, to appear in Proceedings of the 45th IEEE Conference on Decision and Control. San Diego, CA.

Wang, S. H., \& Davison, E. J. (1973). On the stabilization of decentralized control systems. IEEE Transactions on Automatic Control. 18(5), 473-478. 